\title{
In-Flight Subsonic Lift and Drag Characteristics Unique to Blunt-Based Lifting Reentry Vehicles
}

\author{
Edwin J. Saltzman* \\ NASA Dryden Flight Research Center, Edwards, California 93523-0273 \\ K. Charles Wang $₫$ \\ Jet Propulsion Laboratory, California Institute of Technology, Pasadena, California 91109 \\ and \\ Kenneth W. Iliffe \\ NASA Dryden Flight Research Center, Edwards, California 93523-0273 \\ DOI: $\underline{10.2514 / 1.18365}$
}

\begin{abstract}
Lift and drag measurements have been analyzed for subsonic flight conditions for seven blunt-based reentry-type vehicles. Five of the vehicles are lifting bodies (M2-F1, M2-F2, HL-10, X-24A, and X-24B) and two are wing-body configurations (the X-15 and the Space Shuttle Enterprise). Base pressure measurements indicate that the base drag for full-scale vehicles is approximately three times greater than predicted by Hoerner's equation for threedimensional bodies. Base drag and forebody drag combine to provide an optimal overall minimum drag (a drag "bucket") for a given configuration. The magnitude of this optimal drag, as well as the associated forebody drag, is dependent on the ratio of base area to vehicle wetted area. Counterintuitively, the flight-determined optimal minimum drag does not occur at the point of minimum forebody drag, but at a higher forebody drag value. It was also found that the chosen definition for reference area for lift parameters should include the projection of planform area ahead of the wing trailing edge (i.e., forebody plus wing). Results are assembled collectively to provide a greater understanding of this class of vehicles than would occur by considering them individually.
\end{abstract}

\section{Introduction}

$\mathbf{C}$ ONTROLLED reentry from low Earth orbit and the upper atmosphere of the Earth continues to be of interest. Motivations include the need for a crew return and rescue vehicle from the International Space Station, a fledgling space tourism industry and its desire for low-cost reusable space access, the potential for future military space operations, and recent plans for human spaceflight and exploration as the space shuttle approaches retirement. In addition, precision entry, descent, and landing (EDL) in support of future planetary missions has become an area of active investigation. Fundamental studies by the NACA and NASA in the late 1950s and early 1960s described three basic methods of atmospheric reentry: ballistic reentry, winged reentry, and wingless lifting-body (semiballistic) reentry. The purely ballistic reentry approach necessitates the use of parachutes or parafoils to land, whereas the lifting-body and wing-body approaches allow the possibility of horizontal runway-type landings, given a sufficiently dense atmosphere such as for Earth. Flight examples of these latter two approaches include the M2-F1, M2-F2, HL-10, X-24A, X-24B, and $\mathrm{X}-15$ vehicles and the Space Shuttle Enterprise (the Enterprise is a nonorbiting flight prototype version of the space shuttle). Most lifting reentry configurations are attractive because of their crossrange and downrange capability and low-speed handling

Presented as Paper 0383 at the 37th AIAA Aerospace Sciences Meeting and Exhibit, Reno, NV, 11-14 January 1999; received 13 March 2006; revision received 22 September 2006; accepted for publication 6 November 2006. Copyright (C) 2006 by the American Institute of Aeronautics and Astronautics, Inc. The U.S. Government has a royalty-free license to exercise all rights under the copyright claimed herein for Governmental purposes. All other rights are reserved by the copyright owner. Copies of this paper may be made for personal or internal use, on condition that the copier pay the $\$ 10.00$ per-copy fee to the Copyright Clearance Center, Inc., 222 Rosewood Drive, Danvers, MA 01923; include the code 0022-4650/ $07 \$ 10.00$ in correspondence with the CCC.

*Senior Research Engineer, P.O. Box 273.

${ }^{\dagger}$ Senior Engineer, Mechanical Systems Division, Mail Stop 300-329. Senior Member AIAA.

${ }^{\ddagger}$ Chief Scientist, Retired, P.O. Box 273. Fellow AIAA. qualities. In addition to their volumetric efficiency, wingless lifting bodies benefit from peak decelerations that are lower than those of ballistic reentry and peak heating rates that are lower than those of winged reentry vehicles. Because of the continued interest in reliable options for reentry in general and the attractive features of lifting reentry in particular, this paper reexamines lift and drag characteristics of the seven aforementioned vehicles during subsonic unpowered flight. A unifying analysis is presented that provides a meaningful basis for understanding the subsonic performance potential of this class of vehicles.

The vehicles examined in this report are the M2-F1, M2-F2, HL10, X-24A, X-24B, and X-15 vehicles and the Space Shuttle Enterprise, which comprise a unique class of aircraft sharing several features in common. These vehicles had lifting reentry shapes and a truncated afterbody forming a blunt base, which resulted in base drag being a significant component of the total drag. In addition, each of these flight vehicles performed routine, unpowered, horizontal landings on either paved or natural surfaces under the control of an onboard human pilot. Furthermore, these vehicles carried highquality, sensor-recorder systems designed for measuring the parameters needed to define lift and drag. Vehicles without these shared features are not addressed herein. The lift and drag data of the vehicles presented herein were obtained during subsonic, unpowered, coasting flights.

The purpose of this study is to assemble flight-measured lift and drag data from these vehicles under common aerodynamic performance parameters or metrics (that is, the data from all seven vehicles are plotted together) in an attempt to unify the results for this class of vehicles. This array of data is intended to collectively yield information that might otherwise escape notice if the vehicles were individually studied. When it is meaningful, selected performance parameters of the subject vehicles are compared with data formats and standards that are based on classical aerodynamic theory and concepts that range from several decades to a century ago (for example, the concepts of Jones, Allen and Perkins, Helmbold, Krienes, Oswald, and, ultimately, Prandtl and Lanchester). Works explicitly used are referenced.

The innovative and intuitive concepts cited earlier were intended for vehicle configurations that are quite different from the subject 
vehicles. For example, the relevant Jones work applied to sharpedged, low-aspect-ratio wings; Allen's and Perkins' related work addressed high-fineness-ratio bodies of revolution; and the concepts of the others applied to moderate-, high-, and even infinite-aspectratio wings. Despite their original purpose or application, several such theoretical relationships and standards have been used herein as a means of organizing and assessing the flight results considered.

This summary of performance metrics is necessarily somewhat limited to comply with journal format requirements. A considerably more comprehensive analysis is available [1], which includes additional metrics, supporting tables, footnotes, six appendices, and more references. Reference [1] and the present summary are intended to provide a useful database and analytical framework with which to compare and evaluate the subsonic aerodynamic performance of new vehicle configurations of the same generic family: low-aspect-ratio lifting reentry shapes with truncated bases. The results can also be used as a first-order design tool to help airframe designers define the outer moldlines of future related configurations as well as assess the predictive techniques used in their design. In addition, this reexamination of lifting reentry vehicles has been prepared and supporting references are cited, so that readers may access the source material and perform independent analyses of these full-scale flight results.

\section{Historical Background}

At a conference held in March of 1958, manned satellites and methods of reentering the Earth's atmosphere were comprehensively studied [2-5]. Three different methods of reentry from Earth orbit were considered and discussed within the first four papers. The three methods were ballistic reentry [2], the wingless lifting body [3], and winged configurations [4]. Reference [3] advocates the lifting body mainly on the basis that its expected hypersonic lift-to-drag ratio of approximately 0.5 would provide a maximum tangential reentry deceleration of approximately $2 \mathrm{~g}$, low enough to allow a pilot to intervene in the control of the vehicle during this portion of the reentry. (For a pure ballistic, nonlifting reentry, the peak tangential deceleration was expected to be approximately $8 g$.)

The first lifting-body concepts included, but were not limited to, very blunt half-cone shapes $[3,5]$. Those concepts later evolved into cone shapes that had higher fineness ratios [6-8], and the capability

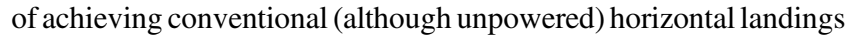
was discussed. Numerous wind-tunnel tests were performed on models of candidate versions of the half-cone shape and shapes with flattened bottom surfaces. In 1962, unpowered horizontal landings and controllable flight were performed with a miniature, lightweight, radio-controlled model of an M2 half-cone configuration [9]. This demonstration was followed by the construction of a lightweight M2 craft large enough to carry a pilot. This unpowered M2-F1 vehicle demonstrated controllable flight and horizontal landings for a maximum lift-to-drag ratio of 2.8 for subsonic flight. The M2-F1 lift, drag, and stability and control characteristics were published in 1965 $[10,11]$.

A heavier and modified version of the M2 shape was built and began flying in 1966. The resulting lift and drag data from subsonic flight were published in 1967 [12]. Other lifting-body configurations (all capable of unpowered horizontal landings) were developed and flight-tested as well. The lift and drag characteristics from subsonic flights have previously been reported for the HL-10 [13], X-24A [14], and X-24B [15] lifting bodies. More information on the evolution and flight testing of the lifting bodies and the evolution of reentry concepts is available $[9,16-19]$.

The M2-F1 and subsequent lifting bodies were not the pioneer vehicles for performing unpowered ("dead-stick") landings, but they were the first vehicles with very low aspect ratios (less than 1.5) to routinely land without power. The early rocket-powered research vehicles (the X-1, X-2, and D-558-II aircraft) were also designed for unpowered landings, but they had higher aspect ratios (between 6.0 and 3.6). Later, the X-15 hypersonic research aircraft, which had a published aspect ratio of 2.5 (between those of the early rocketpowered vehicles and the lifting bodies), made routine dead-stick landings. Confidence in the X-15 aircraft being able to land unpowered [20] was based on the successful experience of the earlier rocket-powered aircraft that had the higher aspect ratios and on a series of special landing investigations using low-aspect-ratio fighter-type airplanes [21]. This study investigated subsonic approach and landings at lift-to-drag ratios of 2 to 4 and used extended gear and speed brakes to increase the drag. Lift and drag data for the X-15 aircraft have previously been published [20,22].

Despite the success of the X-15 unpowered landing experience, the early planning for the space shuttle considered "pop-out" auxiliary engines to ensure safe horizontal landings. However, further consideration of the X-15 and lifting-body experience rendered landing engines for the space shuttle as an unnecessary weight and payload penalty [23]. The space shuttle ultimately was designed to make unpowered landings, and thus became the heaviest of the reentry-type vehicles to use routine dead-stick landings. (The Enterprise was 120 times the weight of the M2-F1 vehicle.) The lowspeed lift and drag characteristics of the Enterprise have previously been published [24]. Results have been reported for the Enterprise with and without a tailcone [24]. Only the truncated configuration (that is, without a tailcone) is subjected to the same tools of analysis that are used on the other six vehicles. Slight use is made of results from the Enterprise (with the tailcone attached) when they reveal a finding that merits documentation.

In recent years, lifting reentry vehicles have been proposed for rescue missions from space $(\mathrm{X}-38)$ and to serve as reusable launch vehicles (X-33, X-34, X-37). These vehicles have much in common with the lifting bodies described herein and, if aspect ratio is somewhat increased, with the X-15 aircraft and the Enterprise. This paper presents the subsonic lift and drag characteristics of the M2-F1, M2-F2, HL-10, X-24A, X-24B, and X-15 vehicles and the Space Shuttle Enterprise under unifying performance parameters and formats, with the intent of aiding the definition of exterior moldlines of future candidate reentry vehicles that perform horizontal landings.

As was mentioned in the Introduction section, some of the unifying metrics depend on borrowed concepts and standards that are several decades old and were originally intended for application on winged vehicles of high or moderate aspect ratio. The authors acknowledge that some readers may disagree with how the borrowed concepts and standards are applied herein. The formats, concepts, and standards that have been used, and the information that may be derived therefrom, are offered as a beginning in the quest for understanding the general nature of the subsonic lift and drag for this unique class of vehicles.

\section{Methods of Analysis and Nomenclature}

This section assembles methods and metrics (performance parameters) used in the analysis of the subject lift and drag data. The primary metrics of aerodynamic performance used herein include lift-curve slope; a modified Oswald lifting-efficiency factor; maximum lift-to-drag ratio; and for minimum drag analysis, equivalent parasite drag area, equivalent skin-friction coefficient, base pressure coefficient, base drag coefficient, and forebody drag coefficient. This list of metrics is not considered all-inclusive; however, it represents those parameters that most directly define lift capability (lift-curve slope and Oswald lifting-efficiency factor). Likewise, lift-to-drag ratio and the other drag factors reliably define subsonic downrange and lateral range capability. Additional lift-drag factors applied to these same vehicles are presented in [1], which is a publishing venue that is less confined in length. This section also defines the nomenclature as the various metrics are introduced.

\section{Lift-Curve Slope}

Trimmed lift-curve slope data for the subject vehicles are compared with potential flow standards for finite-span wings. The most exact theoretical solution for unswept, rectangular wings at incompressible conditions is considered to be that derived by Krienes [25]. Krienes's relationship for lift-curve slope $C_{L_{\alpha}}$ and aspect ratio $A$ is well represented by the following relationship from Helmbold 
[26], as expressed by Polhamus [27], where $C_{L_{\alpha}}$ is represented in $\mathrm{rad}^{-1}$ :

$$
C_{L_{\alpha}}=\frac{2 \pi A}{\sqrt{A^{2}+4}+2}
$$

As $A$ approaches infinity, $C_{L_{\alpha}}$ approaches $2 \pi$. At the lowest aspect ratios, Eq. (1) merges with the linear relationship of Jones [28]:

$$
C_{L_{\alpha}}=\pi A / 2
$$

Equations (1) and (2) represent lift caused by circulation. Neither of these relationships account for leading-edge vortex lift, such as is developed by highly swept delta wings [29], nor lift generated by vortices resulting from crossflow over the forebody [30-32]. The relationships represented by Eqs. (1) and (2) are each oblivious to the effects of trim. Although all seven vehicles violate the limitations of Eqs. (1) and (2), as any aircraft during trimmed conditions generally does, these equations are considered to be rational standards for evaluating the relative lifting capability of the subject configurations. The slopes for the lift curves of the present study were obtained over the lift coefficient range extending from the lowest lift coefficient achieved for a given maneuver to a lift coefficient somewhat greater than that required to obtain maximum lift-to-drag ratio.

\section{Maximum Lift-to-Drag Ratio}

The maximum lift-to-drag ratio $(L / D)_{\max }$ achieved by each of the subject vehicles at subsonic speeds is presented as a function of $b^{2} / A_{w}$ (i.e., span-squared, divided by wetted area). This form of aspect ratio is referred to as the "wetted aspect ratio" [33]. This presentation includes a reference framework consisting of a family of curves representing constant values of equivalent skin-friction coefficient or equivalent viscous-drag coefficient $C_{F_{e}}$, which is a form of minimum drag coefficient $C_{D_{\min }}$ (which includes both forebody and base drag). Thus, if

$$
C_{D_{\min }}=\frac{D_{\min }}{\bar{q} S}
$$

where $\bar{q}$ is dynamic pressure and $S$ is the reference area, then

$$
C_{F_{e}}=C_{D_{\min }} \frac{S}{A_{w}}
$$

Although $C_{F_{e}}$ is called the "equivalent skin-friction coefficient," the important word is equivalent, because $C_{F_{e}}$ is composed of base drag, separation losses, interference drag, protuberance drag, and other losses, in addition to skin friction. The family of reference curves is analogous to that employed in [34], and the curves are defined by the following often-used expression from [35]:

$$
(L / D)_{\max }=\frac{1}{2} \sqrt{\frac{\pi A \varepsilon}{C_{D_{\min }}}}
$$

where the Oswald lifting-efficiency factor $\varepsilon$ [36] is modified as demonstrated in [37], as follows:

$$
\varepsilon=\frac{\left(C_{L}-C_{L_{\min }}\right)^{2}}{\pi A\left(C_{D}-C_{D_{\min }}\right)}
$$

\section{Minimum Drag of the Vehicle}

Minimum drag is considered in several formats. If the lift coefficient and drag coefficient are based on vehicle planform reference area, the minimum drag coefficient can be defined as in Eq. (3). The discussion on maximum lift-to-drag ratio also revealed that another metric for minimum drag coefficient is the equivalent skin-friction coefficient [Eq. (4)], which is obtained by basing the minimum drag coefficient on the wetted area $A_{w}$. The wetted area for each vehicle is considered to be the wetted area of the respective forebody, which includes the body and wings or fins, and thus is the sum of all outer moldline surfaces ahead of an associated base or trailing edge.

Another format for comparing minimum drag for various configurations is the equivalent parasite drag area $f$. This metric is related to Eq. (3) but eliminates controversy regarding the choice of reference area by being defined as follows:

$$
f=C_{D_{\min }} S=\frac{D_{\min }}{\bar{q}}
$$

Thus, $f$ is the minimum drag divided by the freestream dynamic pressure. Use of equivalent skin-friction coefficient $C_{F_{e}}$ [Eq. (4)] and equivalent parasite drag area $f$ [Eq. (7)] is common among aircraft designers. An early example of their use is given in [38].

Minimum drag has previously been represented as three metrics: $C_{D_{\min }}$, in which the reference area is the vehicle planform area $S$, which is sometimes defined subjectively; $C_{F_{e}}$, in which the reference area is the forebody wetted area $A_{w}$, which can be defined objectively and accurately; or as $f$, in which the reference area is eliminated as a factor. Despite any confusion that might result from such names as "equivalent skin-friction coefficient" and "equivalent parasite drag area" (which have been commonly used for many years), each of the metrics presented earlier for minimum drag should be understood to include all losses caused by the forebody (that is, body plus fins, protuberances, control surfaces, and, if applicable, wings) as well as the drag caused by all base surfaces. Mathematically speaking, the following exists:

$$
C_{D_{\min }}=C_{D_{\text {fore }, S}}+C_{D_{b}} \frac{A_{b}}{S}
$$

and

$$
C_{F_{e}}=C_{F_{e, \text { fore }}}+C_{D_{b}} \frac{A_{b}}{A_{w}}
$$

where $C_{D_{\text {fore }, S}}$ is the forebody drag coefficient referenced to $S, C_{F_{e, \text { fore }}}$ is the equivalent skin-friction coefficient caused by the forebody only, $C_{D_{b}}$ is the coefficient of base drag (here based on reference area $A_{b}$, which is the base area).

\section{Minimum Forebody Drag}

Significant excess forebody drag exists, in addition to the drag caused by skin friction. One method to quantify the excess forebody drag is to compare the measured minimum drag of a vehicle with the sum of the measured base drag and the calculated skin-friction drag for completely attached, turbulent, boundary-layer flow. The difference that results from this comparison represents losses from multiple sources, which are designated as "excess forebody drag." The calculated, idealized sum of the base drag and skin-friction drag for each vehicle is obtained from the following:

$$
C_{F_{e}}=C_{F}+\left|C_{P_{b}}\right| \frac{A_{b}}{A_{w}} c
$$

where $C_{F}$ is the turbulent boundary-layer skin-friction coefficient (calculated) of the forebody and $c$ is a base pressure profile factor.

The values of $C_{F}$, representing idealized forebody losses, have been calculated for each of the vehicles at the various flight conditions; adjusted for compressibility effects by the reference temperature method as applied by Peterson [39]; and adjusted for form factor (three-dimensionality) by the coefficient 1.02 , as recommended for conical flow [40]. The value of $C_{F}$ used to calculate the reference curves presented herein is 0.0023 , which is the average value of $C_{F}$ for the various vehicles at the flight conditions reported herein. The constant $c=0.92$ is a base pressure profile factor and its origin is explained in [1].

\section{Base Pressure Coefficients}

Flight-measured base pressure coefficients, base pressure coefficients derived from published incremental drag attributed to 
the base, and estimated base pressure coefficients derived from those of a closely related, afterbody-base configuration are compared with two analytical equations developed by Hoerner [32]. These equations were derived from wind-tunnel experiments of small-scale models. Hoerner's equation for three-dimensional axisymmetric bodies of revolution is as follows:

$$
-C_{P_{b}}=\frac{K}{\sqrt{C_{D_{\text {fore }, b}}}}
$$

where $K=0.029$ and $C_{D_{\text {fore }, b}}$ is referenced to base area $A_{b}$. Hoerner's equation for quasi-two-dimensional base flow conditions that generate the well-known Kármán vortex street is

$$
-C_{P_{b}}=\frac{0.135}{\sqrt[3]{C_{D_{\text {fore }, b}}}}
$$

\section{Lift and Drag Coefficients}

The flight-measured lift and drag coefficients $C_{L}$ and $C_{D}$ for all seven vehicles were obtained by the accelerometer method [1ㅗㄴ 42$]$. The relationships for unpowered, gliding flight are

$$
\begin{aligned}
& C_{L}=\left(a_{n} \cos \alpha+a_{l} \sin \alpha\right) \frac{W}{\bar{q} S} \\
& C_{D}=\left(a_{n} \sin \alpha-a_{l} \cos \alpha\right) \frac{W}{\bar{q} S}
\end{aligned}
$$

where $a_{n}$ and $a_{l}$ are the normal and longitudinal accelerations, $\alpha$ is the angle of attack, $W$ is the vehicle weight, $\bar{q}$ is the freestream dynamic pressure, and $S$ is the reference area.

\section{Data Uncertainty}

The accurate determination of lift and drag characteristics from flight data requires high-quality sensors and careful attention to detail in sensor calibration and use. In general, lift and drag determination is most sensitive to error in the measurement of thrust, longitudinal and normal acceleration, angle of attack, static pressure, Mach number, vehicle weight, and an accounting of control deflections. For the seven vehicles considered herein, thrust is not a factor, because the data were obtained during coasting flight; thus, a major source of uncertainty is avoided. Some of the problems associated with the measurement of these quantities, and their relative importance, are discussed in [42].

Uncertainty information has been published for four of the subject aircraft: M2-F1, M2-F2, HL-10, and X-15 vehicles. A summary of the available uncertainty data is shown in Table 1. Background information pertaining to the origins of the data in this table is given in [1]. The right-hand column shows the uncertainty in base pressure coefficients $C_{P_{b}}$.

These uncertainties represent the square root of the sum of the squares for each of these coefficients when plotted as individual data points. Because these coefficients (as used herein) are obtained from curves faired through numerous data points, the uncertainty of the coefficients resulting from faired data and other metrics should be significantly smaller than those shown in Table 1.

Corresponding uncertainties are not available for the X-24A and X-24B lifting bodies and the Space Shuttle Enterprise; however, air

Table 1 Data uncertainties

\begin{tabular}{lccc}
\hline \hline Vehicle & $\Delta C_{L} / C_{L}, \%$ & $\Delta C_{D} / C_{D}, \%$ & $\Delta C_{P_{b}} / C_{P_{b}}, \%$ \\
\hline M2-F1 & \pm 3.0 & \pm 5.5 & \pm 7.0 \\
M2-F2 & \pm 1.7 & \pm 3.2 & Not available \\
HL-10 & \pm 3.2 & \pm 3.9 & Not available \\
X-15 & \pm 4.3 & \pm 3.9 & \pm 6.4 \\
\hline \hline
\end{tabular}

data system calibration procedures similar to those used on the other four vehicles are known to have been used on these three. In addition, lift and drag were obtained by the accelerometer method for all seven vehicles. Although Table 1 cannot be established as representing the uncertainties for the latter three vehicles, expecting their uncertainties to be relatively close to those listed is not unreasonable.

\section{Results and Discussion}

The primary results of this summary study are presented and discussed under three subheadings: "Lift-Curve Slope," "Maximum Lift-to-Drag Ratio," and several metrics of "Minimum Drag." These and other aerodynamic performance metrics, as applied to the subject vehicles, have been reported in [1] in significantly greater detail. Formats for collectively presenting the data were chosen in the hope that one or more formats will yield a greater understanding of the data than would likely occur by individually studying the subject vehicles.

\section{Lift-Curve Slope}

This section attempts to unify the lift capabilities of the seven flight vehicles previously discussed. The lift-curve slope data for subsonic flight of these vehicles have been assembled from [10,12-15,22,24]. Data were obtained during gradual pushover/pullup maneuvers (consequently trimmed for the respective maneuvers) over a range of lift coefficients extending to somewhat greater than that required to achieve the maximum lift-to-drag ratio. These data are compared with generic wind-tunnel model data and to theory for very low and moderately low aspect ratios. Figure 1 shows three-view drawings of each of the seven vehicles and the M2-F3 lifting body. Major dimensions for each vehicle are presented in Table 2 .

Figure 2 shows lift-curve slope results for the seven subject vehicles, plotted as a function of aspect-ratio. The often-used Prandtl-Glauert factor $\left(1-M^{2}\right)^{0.5}$ has been applied to both the ordinate and abscissa functions, because data for the various vehicles were obtained at different subsonic Mach numbers. Wind-tunnel model data for simple generic lifting-body shapes are also included $[43,44]$. Figure 2 also shows the relationships of $C_{L_{\alpha}}$ to aspect ratio as defined by Helmbold [Eq. (1)] and, for the lowest aspect ratios, the linear relationship of Jones [Eq. (2)]. Neither of these relationships accounts for lift from crossflow over the bodies or from vortices generated by sharp, highly swept leading edges. Stated another way, Eqs. (1) and (2) apply when the flow does not separate from leading or swept lateral edges (that is, these equations represent lift generated by circulation).

The lift-curve slopes for each of the flight vehicles were expected to occur below the Jones and Helmbold relationships, which represent maximum efficiency for medium- or low-aspect-ratio configurations that obtain their lift from circulation. However, the slope value for the M2-F2 is above the theoretical curves, and the slope for the X-15 is relatively high (i.e., between the Jones and Helmbold curves).

The data from the generic model shapes (solid symbols) [43, 44$]$ suggest that the M2-F2 slope occurring above the theoretical curves should not be considered to be an anomaly. The reason that the M2F2 vehicle and the slender model shapes (that is, those having aspect ratios less than 1.0) have relatively high lift-curve slopes may be related to well-developed forebody vortices caused by crossflow, as reported in [30-32]. The half-cone shapes, having lateral edges with a small radius, were expected to produce vortex lift. However, the elliptical cone shape with the most slender planform (the lowest aspect ratio) also had a high slope compared with potential theory. Thus, the conjecture that well-developed vortices (resulting from body crossflow) may provide an extra component of lift is afforded credence, even if sharp lateral edges are absent.

Because of this evidence that crossflow (counter-rotating vortex pair) effects may significantly contribute to the lift of the slender forebody portions of lifting bodies, considering that the forebodies of the X-15 aircraft and the Enterprise may likewise generate significant amounts of crossflow lift is appropriate. For these winged 


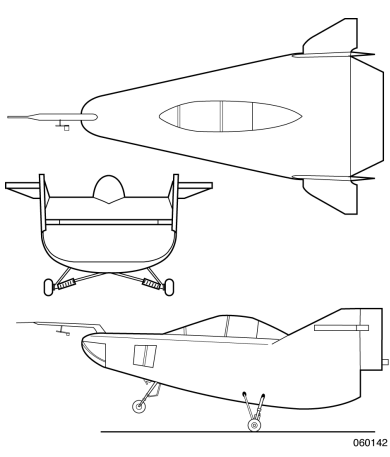

a) M2-F1 vehicle, $b=14.17 \mathrm{ft}$

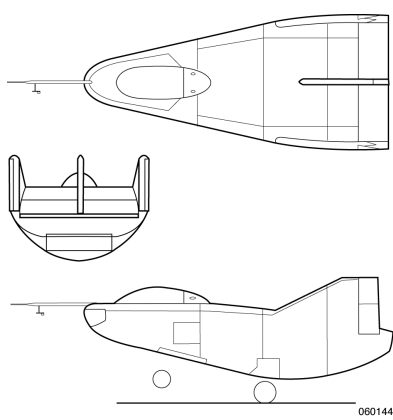

c) M2-F3 vehicle, $b=9.95 \mathrm{ft}$

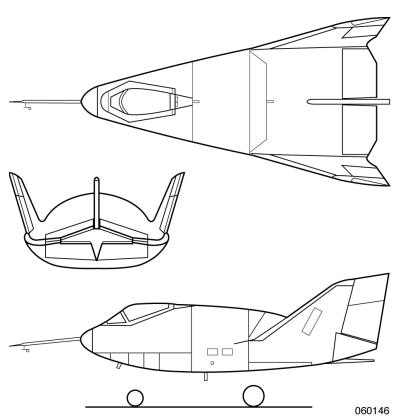

e) X-24A vehicle, $b=13.63 \mathrm{ft}$
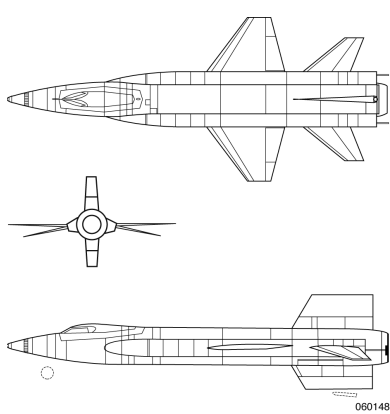

g) $\mathrm{X}-15$ vehicle, $b=22.36 \mathrm{ft}$

Fig. 1 Three-view drawings of the subject vehicles; $b$ indicates the span in feet.

vehicles, therefore, the forebody planform area and the wing area projected to the vehicle centerline are considered to be the reference area. Because the revisions of reference area for the X-15 and space shuttle vehicles are a departure from convention, and because two separate concepts are involved, additional discussion and supporting data are justified. First, inclusion of the forebody planform area with the wing-panel area is justified in part, on the basis of the crossflow lift experienced by lifting bodies $[30-32,43,44]$. In addition, Appendix D of [1] cites fuselage normal force data obtained in-flight from two aircraft (the X-1 and the X-15) that establish that fuselage lift is significant. The second concept, which rejects fuselage planform area aft of the wing trailing edge for inclusion as reference area, conforms to [28] (pgs. 59 and 63), which postulates that for pointed shapes, "sections behind the section of maximum width develop no lift." This theory, and flight data from the X-1 airplane [45], also shown in Appendix D of [1] , taken together, constitute the rationale for this second concept.

As noted earlier, the lift-curve slope data from the half-cone [43] and elliptical cone [44] models tend to confirm the M2-F2 flight results, which exceed the Jones relationship. The values for the elliptical cone models at aspect ratios greater than 1, however, have lift-curve slopes that are significantly lower than both the Helmbold and Jones relationships [Eqs. (1) and (2), respectively]. For the elliptical cone shapes having the highest aspect ratios (that is, clearly nonslender), a lift component caused by circulation dominates and some degree of crossflow additionally exists; whereas at the lowest aspect ratios, the crossflow component of lift is more dominant $[31,32]$.

The model data of $[43,44]$ represent untrimmed conditions; consequently, their lift-curve slopes are expected to be optimistic. It is not surprising, then, that the lift-curve slopes for the five lifting bodies obtained at full scale during trimmed flight form a crude band arrayed approximately 12 to $15 \%$ below the small-scale, untrimmed, elliptical cone model results. On the other hand, it is clear that the X15 and shuttle vehicles (which had wings and lifting-body-like forebodies) benefited significantly from the combination of both lifting components.

The degree of order, or coalescence, of the lift-curve slope data from the several vehicles was achieved only after revising some of the reference areas and span dimensions from those previously published with the original lift-curve data. For the lifting bodies, the physically meaningful reference area should include all planform area projected onto the longitudinal-lateral plane, including the projected area of canted tip or side fins. Improved order was also provided by adjusting the data for compressibility effects. Dimensions given in $[\underline{46}, \underline{47}]$ were used to revise those published with the original lift-curve data for the M2-F2 and X-24A vehicles, respectively.

\section{Maximum Lift-to-Drag Ratio}

Figure 3 shows maximum lift-to-drag ratio as a function of the ratio of span-squared to wetted area for each of the vehicles in subsonic flight. This format is commonly used by designers of conventional subsonic aircraft, because at subsonic speeds, air vehicle efficiency is most directly influenced by span and wetted area. Reference [33] refers to this abscissa function as the "wetted aspect ratio."

For the lifting bodies, the X-15 vehicle, and the Enterprise, all of which have significant amounts of base drag, recognizing the "base" effects by assigning base drag to the equivalent skin-friction coefficient parameter $C_{F_{e}}$ is necessary. Consequently, Fig. 3 also shows a reference framework consisting of a family of constant values of $C_{F_{e}}$, as employed by [34]. This family of curves is derived from the often-used expression that relates maximum lift-to-drag ratio to the minimum drag coefficient (here, expressed as $C_{F_{e}}$ ), aspect ratio, and the lifting-efficiency factor $\varepsilon$ [Eq. (5)]. The range of the family of $C_{F_{e}}$ curves shown in Fig. 3 covers the range of values experienced by the subject vehicles. Thus, the format used will accommodate this class of vehicles for which minimum drag consists of a large component of base drag as well as friction drag. A liftingefficiency factor $\varepsilon$ of 0.6 was assigned to these curves, because this value is approximately the average for the subject vehicles as a group. The dashed curve for the equivalent skin-friction coefficient is included, because it represents a nominally clean modern aircraft that does not have a truncated body and is constrained to an $\varepsilon$ factor of 0.6. Note in Fig. 3 that when the Enterprise had its blunt base covered with a tailcone, the maximum lift-to-drag ratio increased to 7.5 , 
Table 2 Physical characteristics of the vehicles

\begin{tabular}{|c|c|c|c|c|c|c|c|c|c|}
\hline Vehicle & $\underset{\mathrm{ft}}{l \text {, length, }}$ & $\begin{array}{c}b, \text { span, } \\
\mathrm{ft}\end{array}$ & $\begin{array}{c}A, \text { aspect } \\
\text { ratio }\end{array}$ & $\begin{array}{c}S, \text { reference } \\
\text { area, } \mathrm{ft}^{2}\end{array}$ & $\begin{array}{c}A_{w}, \text { wetted } \\
\text { area, } \mathrm{ft}^{2}\end{array}$ & $\begin{array}{c}A_{b}, \text { base } \\
\text { area, } \mathrm{ft}^{2}\end{array}$ & $\begin{array}{c}W \text {, weight, } \\
\mathrm{lb}\end{array}$ & $M$, Mach range & $\begin{array}{c}\text { Fineness } \\
\text { ratio- }\end{array}$ \\
\hline M2-F1 & 20.00 & 14.17 & 1.318 & 152.4 & 431.0 & 30.84 & 1250 & 0.15 & 2.50 \\
\hline M2-F2 & 22.20 & 9.95 & 0.619 & 160.0 & 459.0 & 22.51 & 6000 & 0.45 to 0.62 & 2.94 \\
\hline $\mathrm{X}-24 \mathrm{~A}$ & 24.50 & 13.63 & 0.953 & 195.0 & 590.0 & 11.78 to 25.36 & 6360 & 0.50 & 2.73 \\
\hline$X-24 B$ & 37.50 & 19.14 & 1.108 & 330.5 & 948.4 & 18.79 to 38.05 & 8500 & 0.50 to 0.80 & 3.93 \\
\hline$X-15$ & 49.50 & 22.36 & 1.629 & 307.0 & 1186.0 & 33.0 & 15,000 & 0.65 to 0.72 & 7.18 \\
\hline
\end{tabular}

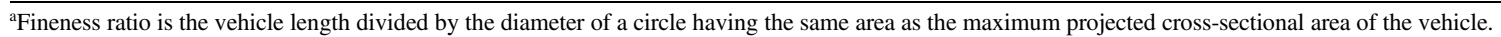

relatively close to the dashed curve. The tailcone partially qualifies this configuration as approximating "nominally clean." However, the intentionally roughened surface of the Enterprise simulating a thermal protection system obviously violates nominally clean requirements. As noted in [1], the tailcone did not contribute to lift; hence, the increase in maximum lift-to-drag ratio can only be attributed to a significant reduction in minimum drag.

The highest values of maximum lift-to-drag ratio at subsonic conditions for five of the blunt-based vehicles and their collective relationship to the reference framework of curves form an array [a band of $(L / D)_{\max }$ over a range of $b^{2} / A_{w}$ ] that should be a useful reference source with which to relate future reentry-type vehicles. A fairing through this data band (as related to the dashed curve) would indicate that this class of vehicles has maximum lift-to-drag ratios that are approximately $55 \%$ of those for nominally clean vehicles, having an $\varepsilon$ value of 0.6 , without truncated bodies (that is, the dashed curve) for a given aspect ratio. The M2-F1 and HL-10 lifting bodies, which are less efficient, should be no less useful to the degree their apparent lesser efficiency is more fully understood.

\section{Minimum Drag}

Minimum drag is presented in several formats to better understand which components are dominant and to reveal the relationship of

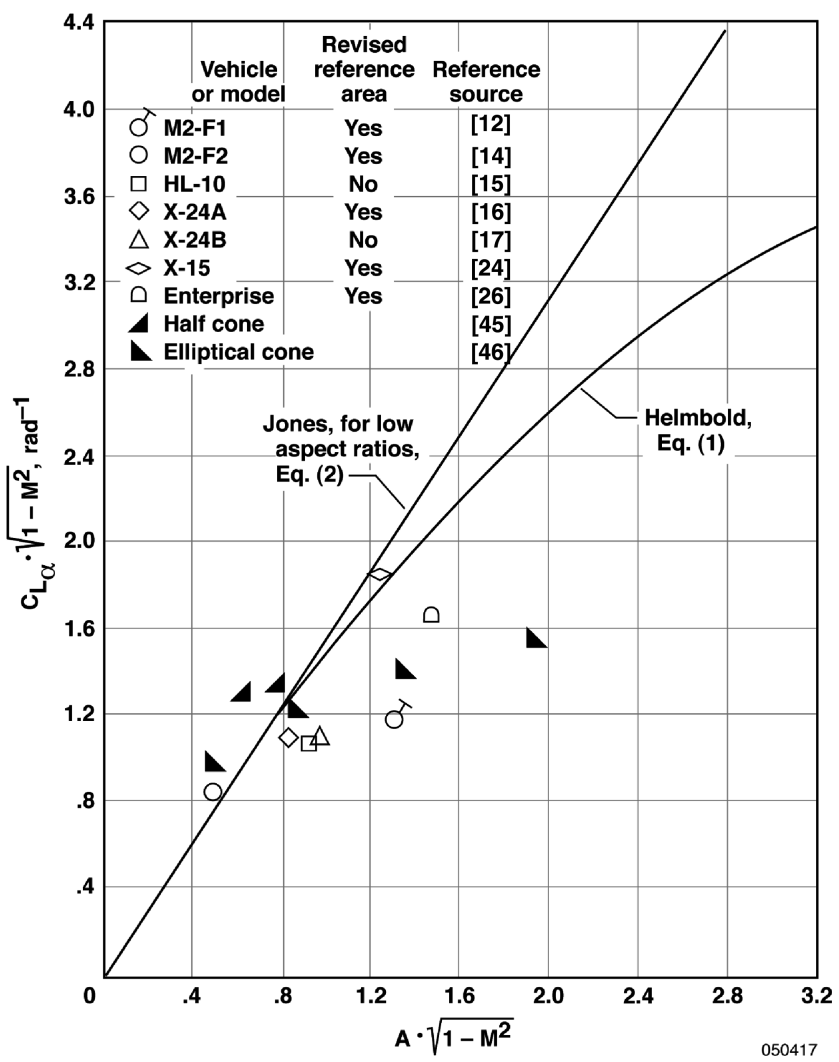

Fig. 2 The relationship of lift-curve slope with aspect ratio as obtained in-flight from generic models and from the theories of Jones and Helmbold (Krienes). forebody and base drag. The metrics used, as defined earlier, include equivalent skin-friction coefficient $C_{F_{e}}$ and equivalent parasite drag area $f$; as previously mentioned, these forms of minimum drag include both base and forebody drag. Base drag is defined for each vehicle (using measurements for five of the vehicles and estimates for the X-24A and X-24B vehicles) to allow separation of base drag and forebody drag components. The data from the vehicles are collectively presented in graphic formats to provide a greater understanding than would likely be achieved by studying the vehicles individually.

Although the revised reference areas are believed to be a rational improvement over the areas that they replace (as noted in the "LiftCurve Slope" section), the format chosen here for graphically presenting the minimum drag eliminates the often arbitrary conventional reference area as a factor. Reference [38] and subsequent others have avoided the concern about reference area definition by multiplying the minimum drag coefficient by the reference area to define an equivalent parasite drag area $f$, as shown in Eq. (7).

Figure 4 shows the equivalent parasite drag area for each of the subject vehicles as a function of total wetted area. The range of equivalent parasite drag area for the subject vehicles is quite large, from 6.5 to $164.0 \mathrm{ft}^{2}$. The total wetted area for each vehicle is defined as all outer moldline or external surface areas ahead of a blunt base or any trailing edge. The definition thus assumes that the flow is attached over these surfaces. Separated regions ahead of the base, interference effects, vortex flow ahead of the base, and negative

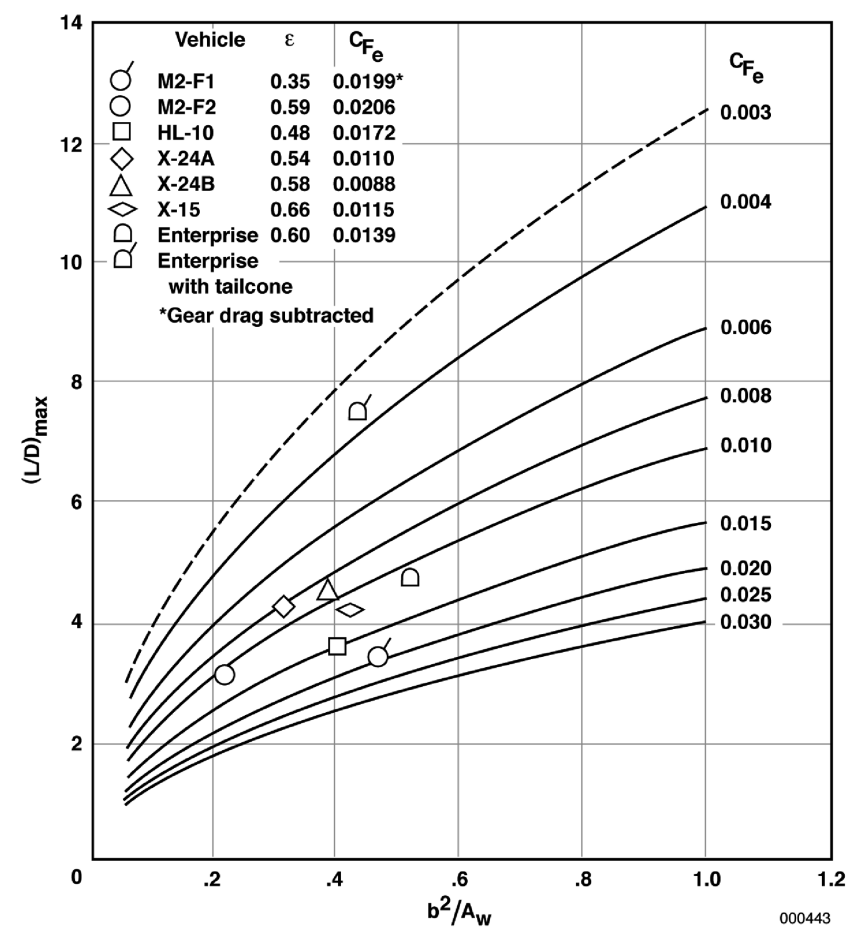

Fig. 3 The relationship of the maximum lift-to-drag ratio to wetted aspect ratio. The family of curves, at constant values of $C_{F_{e}}$, is derived using Eqs. (4) and (5). 


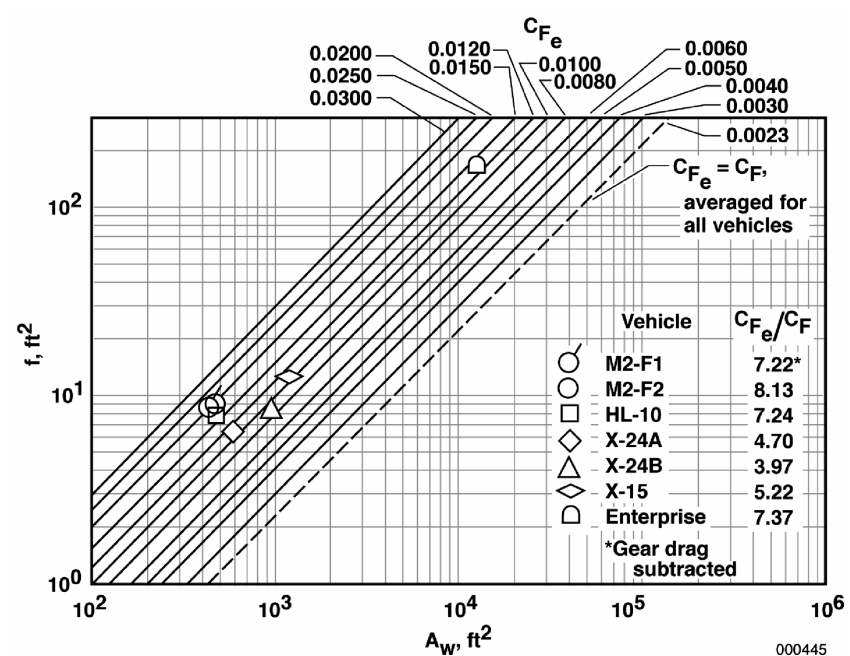

Fig. 4 The relationship of equivalent parasite drag area and equivalent skin-friction coefficient to total wetted area.

pressure coefficients over the base or aft sloping surfaces each represent drag increments in excess of the viscous drag generated by the actual wetted surfaces. Hence, this drag metric defines the sum of the drag sources (excluding lift) that include friction drag for turbulent flow conditions as well as drag components in excess of friction. Because even an ideal body will have friction drag, this metric is labeled as a "parasite" factor because the metric includes such parasitic losses.

The equivalent parasite drag area can also be interpreted in terms of an equivalent skin-friction coefficient $C_{F_{e}}$ by noting the location of a datum point for a given vehicle relative to the family of constant equivalent skin-friction lines (Fig. 4). The equivalent skin-friction coefficient is, of course, another metric that reveals the degree to which measured minimum drag of a vehicle exceeds the ideal minimum drag (that is, the skin-friction drag over the wetted area). The average subsonic skin-friction coefficient over wetted areas for all seven vehicles, assuming flat-plate, turbulent boundary-layer flow (adjusted by a form factor of 1.02) at flight Reynolds numbers, is $C_{F}=0.0023$, which can also be considered as a reference value of $C_{F_{e}}$ (see the dashed line in Fig. 4).

A cursory summary of the data shown in Fig. $\underline{4}$ can be stated as follows:

1) The early generations of lifting bodies, the M2 and the HL-10 vehicles, have equivalent skin-friction coefficients between 0.0170 and 0.0200 (in contrast to the average value of skin friction for all seven vehicles for turbulent flow, 0.0023).

2) For the $X-24 A$ and $X-15$ vehicles, the corresponding equivalent skin-friction coefficients are approximately 0.011 .

3) The X-24B vehicle, the last of the lifting bodies, has an equivalent skin-friction coefficient slightly less than 0.009 .

4) The wetted surfaces of the Enterprise were purposely roughened to simulate the thermal protection tiles of operational vehicles to follow. In addition, this vehicle has a very large base area. Consequently, the Enterprise equivalent friction coefficient of approximately 0.014 is understandably higher than the three lowest values and occupies the median position in the array of coefficients for the subject vehicles.
Note that the range of the equivalent skin-friction coefficients for each of the seven vehicles, from approximately 0.009 to 0.020 , is from four to slightly more than eight times the skin-friction drag that would occur from an attached turbulent boundary layer alone (see the tabular values for each vehicle, listed in Fig. 4).

\section{Base Pressure Coefficients}

Hoerner [32] compiled base pressure data from projectiles, fuselage shapes, and other small-scale three-dimensional shapes and derived therefrom an equation that related the base drag and base pressure coefficients to the forebody drag of the respective bodies [Eq. (11)]. Reference [32] also includes an equation that describes the analogous relationship for quasi-two-dimensional shapes that shed vortices in a periodic manner, the well-known Kármán vortex street [Eq. (12)]. Base pressure data from some of the subject vehicles will be compared on the basis of the Hoerner relationships and modifications to his equations (using different $K$ values). The search for flight-measured base pressure data for the seven subject vehicles is somewhat disappointing, considering that each of these vehicles has a significant component of base drag. Table 3 shows the results of the literature search.

Note from Figs. $1 \mathrm{~b}$ and $\underline{1 \mathrm{c}}$ that the M2-F3 vehicle is virtually the same as the M2-F2 vehicle. All configurational dimensions are the same except that a centerline upper vertical fin was added to the M2F3 vehicle. For this reason, the unpublished base pressure data from the M2-F3 lifting body are accepted as representative of those of the M2-F2 lifting body. Consequently, the M2-F2 and the M2-F3 lifting bodies will be treated as if they were the same vehicle in the analysis to follow.

Because of Hoerner's convincing demonstration that base pressure is related to forebody drag, comparing the available base pressure coefficients from the subject vehicles to his equations is possible. Figure $\underline{5}$ shows these comparisons. Figure $\underline{5}$ also includes a shaded band for Hoerner's three-dimensional equation that is bounded by numerator coefficients $K$ of 0.09 and 0.10 . By modifying Hoerner's original equation with these $K$ coefficients, the base pressure coefficients from the X-15, the M2-F3, and the space shuttle vehicles (which are obviously three-dimensional) are observed to fall within, or relatively close to, this band.

Figure 5 also shows that the flight data are relatively close to Hoerner's quasi-two-dimensional relationship [Eq. (12)]. The relatively higher (more negative) pressure coefficient from the $\mathrm{X}$ $24 \mathrm{~B}$ vehicle (dark triangle) is caused by the large wedge angle, ahead of the base, formed by the upper and lower flaps that are used for control in pitch. The upper flap was deflected upward approximately $40 \mathrm{deg}$, and the lower flap was deflected downward approximately $28 \mathrm{deg}$. This geometry is known to produce more negative base pressure coefficients [51]. The only measured base pressure data from the X-24B vehicle [48], unfortunately, were obtained with a significantly larger wedge angle than existed for the subsonic control configuration for the X-24B data of this study.

The M2-F1 datum is somewhat unrepresentative of the subject class of vehicles, in that the base region was pressurized to some extent by turning vanes (one on each side, below the rudders). Based on the available flight data, the vehicles considered herein (excepting the data for the M2-F1 vehicle and the X-24B transonic configuration) are best represented by the three-dimensional equation where $K=0.09$ to 0.10 , which means that the base drag of

Table 3 Base pressure sources

\begin{tabular}{|c|c|c|c|}
\hline Vehicle & $C_{P_{b}}$ data & Reference number & Remarks \\
\hline M2-F1 & Yes & [10] & The base region was pressurized by turning vanes. \\
\hline M2-F3 & Yes & Unpublished & The M2-F3 base pressure data were applied to the M2- F2 vehicle drag data. \\
\hline HL-10 & No & & Base drag data have been published, but no explicit base pressure data were found. \\
\hline $\mathrm{X}-24 \mathrm{~B}$ & Yes & [48] & Base pressure coefficients were estimated using Mach 0.8 results (Appendix F of [1]). \\
\hline $\mathrm{X}-15$ & Yes & ["푸] & \\
\hline Space shuttle & Yes & [50] & $\begin{array}{l}\text { Base pressure data from the orbiting Space Shuttle Columbia have been applied } \\
\text { to the drag data from the nonorbiting Enterprise. }\end{array}$ \\
\hline
\end{tabular}



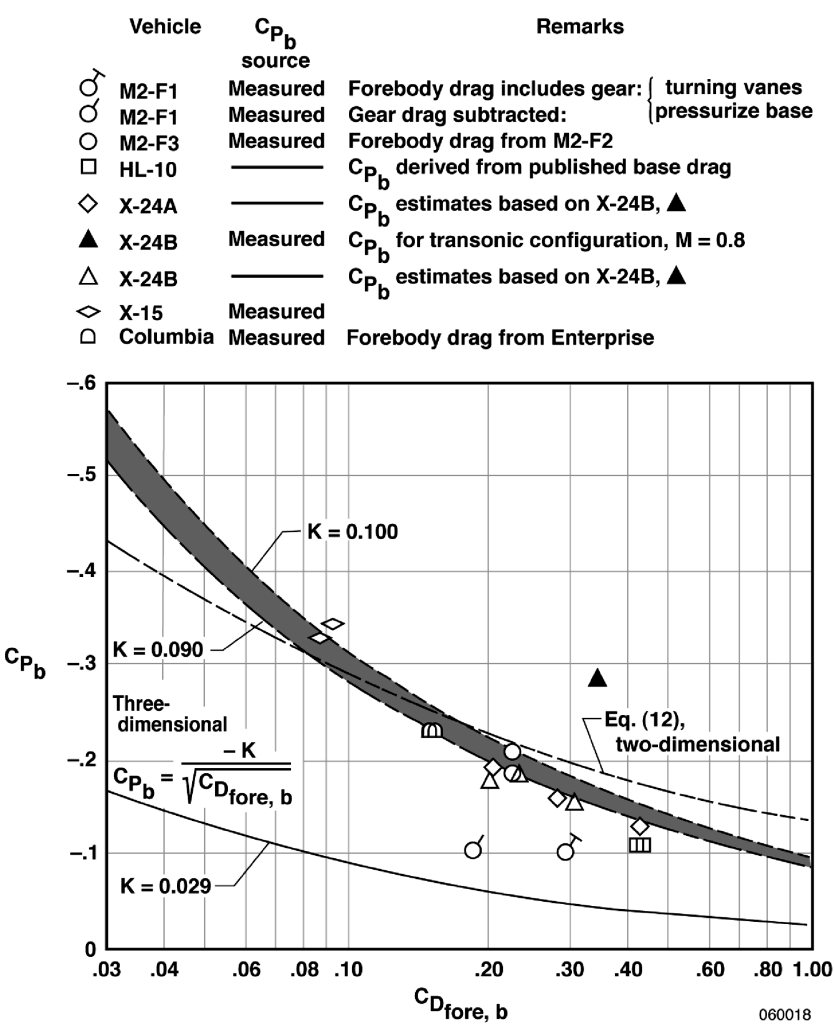

Fig. 5 Comparison of base pressure coefficients for subject vehicles with Hoerner's two-dimensional relationship and with a revised threedimensional equation.

blunt-based large-scale vehicles is much higher than predicted by the original three-dimensional equation. Based on evidence from $[49,52]$ and Fig. 5, subsonic flow separating from a relatively large, sharp-edged, three-dimensional base can be argued to exhibit quasitwo-dimensional characteristics. In either case, the data indicate much more negative base pressure coefficients than the unmodified three-dimensional equation $K=0.029$ would predict.

\section{Optimal Minimal Drag}

Excluding the base pressure data from the M2-F1 and the X-24B vehicles for the reasons already given, the flight data from three other vehicles (M2-F3, X-15, and space shuttle vehicles) are believed to represent the generic blunt-based class of vehicles. More large-scale base pressure and overall minimum drag (and hence forebody drag) data must be obtained in-flight to convincingly demonstrate their relationship. Defining this relationship for three or four values of forebody drag for the same outer moldline shape would be most helpful. Until more flight data are obtained or a superior relationship is developed, the shaded region of Fig. 5, derived from the data of the latter three vehicles, is assumed to be a reasonable representation of the base pressure characteristics for this class of reentry craft. Therefore, a revised version of Eq. (11), where $K=0.10$, has been used to show the dependence of minimum drag on the relative size of the blunt base over a significant range of forebody drag for subsonic conditions.

Figure 6 shows this relationship, in which each of four curves shows how overall minimum drag coefficient varies with forebody drag coefficient for discrete ratios of base area to wetted area (2.5, 5.0, 7.5, and $10.0 \%$ ). The salient feature of these curves is that each has what will be referred to as an optimal region of lowest overall minimum drag coefficient $C_{F_{e}}$. This lowest value is called optimal here, because optimal is more concise and less cumbersome than the repetitious use of lowest minimum or the minimum of the minimum; however, it is acknowledged that the term optimal would usually be used to signify the ideal in which a vastly more comprehensive range of factors that can influence performance is considered.
Note that for the $2.5 \%$ relationship, an optimal region (a drag "bucket") exists near the forebody drag coefficient value of 0.003 . Because these coefficients are based upon the wetted area, and because the smooth-skin turbulent friction coefficient for these Reynolds numbers (in the range of $10^{7}-10^{8}$ ) would be close to 0.002 , a configuration having a base-area-to-wetted-area relationship of $2.5 \%$ can afford only a minute amount of roughness, protuberance, interference, or separation drag over the forebody if the optimal $C_{F_{e}}$ is to be achieved. Conversely, for the high base-area-to-wetted-area relationships, which more closely represent several conceptual reusable launch vehicle and reentry configurations, the optimal $C_{F_{e}}$ (or ift and draget) occurs at significantly higher values of forebody drag coefficient $C_{F_{e \text { fore }}}$.

This characteristic should be of particular interest with regard to some conceptual reusable launch vehicles that have relatively large base-area-to-wetted-area ratios (between 7.5 and 10.0\%). This observation points to the counterintuitive, but welcome, condition that such configurations can afford (in fact, may benefit from) additional forebody drag, in addition to the unavoidable smooth-skin turbulent friction. Thus, surface roughness that may accompany a thermal protection system may actually provide a reduction in overall $C_{F_{e}}$ while increasing the forebody drag, if the upper body is shaped so as to maintain attached, high-energy flow.

Such a reduction would be the result of forebody roughness affecting the growth of the boundary layer from the nose to the edge of the base, which, in turn, affects the level of "vacuum" or suction at the base through a "jet-pump mechanism," as described in [32]. Thus, subject to the curves of Figs. 5 and 6 , forebody roughness adds to the thickness of the boundary layer, thereby reducing the pumping (vacuuming) of the base and reducing the base drag. The drag bucket curves (Fig. 6) are related to those seen in Chapters 3, 6, and 13 of [32] for bodies, nacelles, canopies, and airfoils.

Figure 6 shows the relationship of $C_{F_{e}}$ to forebody drag coefficient for the same vehicles that are represented in Fig. 5. The numbers adjacent to each data symbol (Fig. 6) indicate the basearea-to-wetted-area ratio (in percentage terms) of the respective vehicle at the specific flight condition. From these numbers, in relationship to the curves, note that the data from the vehicles designated by the open symbols (except the M2-F1 and HL-10 vehicles) are in qualitative accord with the semi-empirical curves. As was stated earlier with regard to Fig. 5 , the overall $C_{F_{e}}$ for the M2-F1 vehicle is believed to be lower than the semi-empirical curves suggest, because turning vanes pressurized the base. For the $\mathrm{X}$-24B vehicle in Fig. 6 (the dark triangle), the value of $C_{F_{e}}$ is believed to be high because of the aforementioned large flare angle that produces high windward surface drag and lowers the leeside pressures on the longitudinal control body flaps. This belief is not only supported by data from [51] but also by speed-brake data from the $\mathrm{X}-15$ aircraft (the dark symbol) that represent a comparable flared, or wedge, angle [53].

With reference to the curves in Fig. 6 , analytically determining the minimum, or bucket, value of the equivalent skin-friction coefficient $C_{F_{e}}$ and the associated forebody equivalent skin-friction coefficient $C_{F_{e, f o r e}}$ for a given base-area-to-wetted-area ratio $A_{b} / A_{w}$ is possible. The curves of Fig. $\underline{6}$ are defined by a revision of Eq. ( $\underline{10})$, repeated here:

$$
C_{F_{e}}=C_{F}+\left|C_{P_{b}}\right| \frac{A_{b}}{A_{w}} c
$$

Equation (10) was used to calculate $C_{F_{e}}$ for a case in which no forebody losses existed, except for that of a fully attached, turbulent boundary over a smooth surface (hence, the term $C_{F}$ on the right side of the equation). For the curves shown in Fig. 6, the abscissa values of $C_{F_{e, \text { fore }}}$ are substituted for $C_{F}$ in Eq. (10), as follows:

$$
C_{F_{e}}=C_{F_{e, \text { fore }}}+\left|C_{P_{b}}\right| \frac{A_{b}}{A_{w}} c
$$




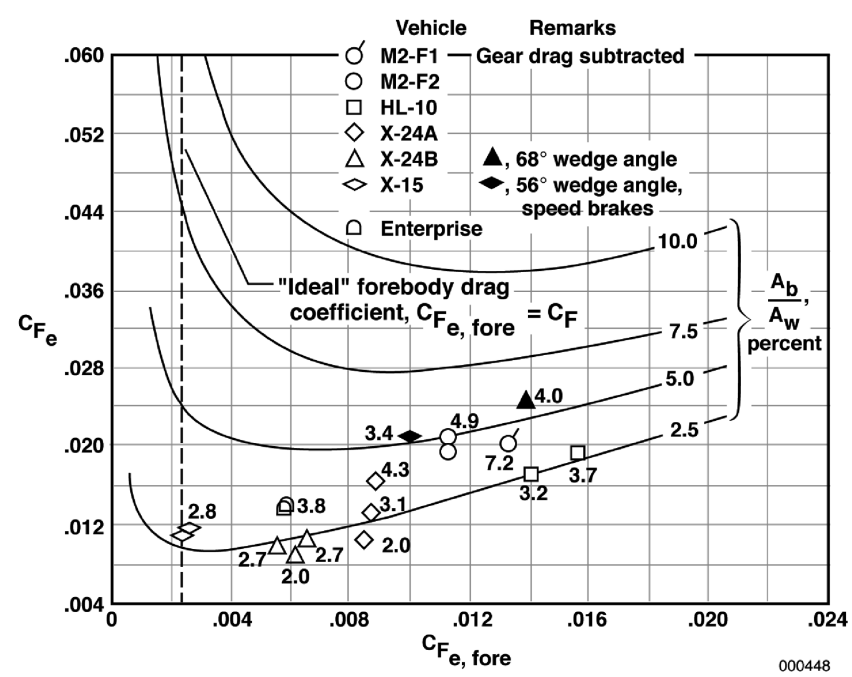

Fig. 6 The relationship of equivalent skin-friction coefficients for the complete vehicle and the forebody.

profile factor, is explained in [1] , and $C_{P_{b}}$ is the expression from [32], Eq. (11):

$$
-C_{P_{b}}=\frac{K}{\sqrt{C_{D_{\text {fore }, b}}}}
$$

where $K=0.10$, based on current analysis. Also,

$$
C_{D_{\text {fore }, b}}=C_{F_{e, \text { fore }}} \frac{A_{w}}{A_{b}}
$$

Substituting Eq. (16) into Eq. (11) and, in turn, Eq. (11) into Eq. (15), gives the following:

$$
C_{F_{e}}=C_{F_{e, \text { fore }}}+\frac{0.10}{\sqrt{C_{F_{e, \text { fore }}}\left(A_{w} / A_{b}\right)}} \frac{A_{b}}{A_{w}} 0.92
$$

To determine the coordinates for the bucket value of $C_{F_{e}}$, the preceding expression is differentiated with respect to $C_{F_{e \text { fore }}}$. Setting the derivative to zero, the minimum $C_{F_{e}}$ value occurs at

$$
C_{F_{\text {,fore }}}=0.1284 \frac{A_{b}}{A_{w}}
$$

Substituting back into Eq. (17), the minimum of $C_{F_{e}}$ for a given base-area-to-wetted-area ratio is

$$
C_{F_{e}}=0.3852 \frac{A_{b}}{A_{w}}
$$

These expressions for optimal values of forebody drag and overall minimum drag coefficient are offered as tools for approximation until more definitive relationships are obtained. The present expressions define the coordinates for minima that are consistent with values obtained graphically from working plots; however, they and the families of curves of Fig. $\underline{6}$ are dependent on multiple assumptions. These assumptions include the validity of the Hoerner equation (11) for three-dimensional flow, the assigned value of $K$ for Eq. (11), and the validity of the base pressure profile factor of 0.92 . Generally, improved values for such curves and the expressions for minima [Eqs. (18) and (19)] can be generated as assumptions become based on a more comprehensive database or as a new superior relationship of the variables is formulated.

\section{Conclusions}

Flight-determined lift and drag characteristics from seven bluntbased lifting-body and wing-body reentry configurations have been compared and related to several standards of aerodynamic efficiency. For lift-curve slope, limited comparisons have been made with wind-tunnel results for generic models and the theoretical relationships of Jones and Helmbold. A summary of major results is as follows:

1) Base pressure coefficient data from the $X-15$, the M2-F3, and the space shuttle vehicles indicate that to represent large-scale flight vehicles, Hoerner's equation relating base pressure to threedimensional forebody drag requires a larger numerator coefficient than Hoerner used. A tentative range of values for the numerator coefficient is from 0.090 to 0.100 , rather than Hoerner's value of 0.029 , which is based on small-scale model data.

2) Evidence exists that subsonic flow separating from a relatively large, sharp-edged three-dimensional base can exhibit quasi-twodimensional characteristics and base pressure coefficients.

3) The nature of the Hoerner base-pressure-to-forebody-drag relationship (regardless of whether the three-dimensional or twodimensional equation is used, or the numerator coefficient value) causes base drag and forebody drag to combine in a counterintuitive way to form an optimal minimum drag (a drag bucket) over a small range of forebody drag. The magnitude of forebody drag coefficient that defines the bucket depends primarily on the ratio of base area to wetted area of the respective vehicle. This means that a vehicle having a large base-area-to-wetted-area ratio and attached upper surface flow may benefit from surface roughness drag (such as that associated with a thermal protection system) at low lifting conditions; this combination of features may provide some favorable compensation for low-fineness-ratio vehicles having a relatively large base.

4) Minimum equivalent parasite drag area values for the vehicles range from 6.5 to $164.0 \mathrm{ft}^{2}$. Division of equivalent drag area by the associated wetted area provided equivalent parasite skin-friction coefficients, ranging from approximately 0.009 to 0.020 (these coefficients include base drag). These minimum equivalent skinfriction values (for retracted landing gear) range from four to slightly more than eight times the skin-friction drag for the attached, turbulent boundary layer alone.

5) Little order existed to the lift-curve slope data when lift coefficient was based on the reference areas used in the reports from which the data were obtained. Application of more representative reference areas (for five of the seven vehicles) and adjustment of the lift-curve slopes for compressibility provided improved order to the data. These data demonstrate that the choice of a physically meaningful (representative) reference area is of major importance.

6) The lift-curve slopes of the five lifting bodies obtained at full scale during trimmed flight form a crude band arrayed approximately 12 to $15 \%$ below untrimmed generic elliptical cone small-scale model results.

7) The chosen definition for a physically meaningful reference area for lift parameters of a lifting body should include all of the planform area projected onto the longitudinal-lateral plane, including the projected area of canted tip or side fins. For wingbody combinations, the fuselage forebody is considered to perform as a lifting body. Therefore, the chosen reference area for these configurations includes the projection of all planform area ahead of the wing trailing edge (that is, forebody plus wing). This concept, which rejects projected body planform area aft of the wing trailing edge, is supported by fuselage pressure distribution measurements from the $\mathrm{X}-1$ research airplane.

8) The M2-F2 data demonstrate that the lift-curve slope of very low-aspect-ratio lifting bodies can exceed the lift-curve slope values represented by the relationships of Jones or Helmbold for aspect ratios less than approximately one. The M2-F2 results are not believed to be an anomaly, because they are afforded credence by generic model results, generic leading-edge vortex lift data from highly swept wings, and crossflow (drag) lift data from bodies of revolution. 
9) Excepting the M2-F1 and the HL-10 vehicles, the remaining five vehicles form an array (a band of the maximum lift-to-drag ratio over a range of the wetted aspect ratio) that should be a useful reference source with which to relate future reentry-type vehicles or reusable launch vehicles. A fairing through this band of data indicates that the maximum lift-to-drag ratio for this class of vehicles, in which the lifting-efficiency factor is limited to 0.6 , is approximately $55 \%$ of those for nominally clean vehicles without truncated bodies for a given wetted aspect ratio.

\section{References}

[1] Saltzman, E. J., Wang, K. C., and Iliff, K. W., "Aerodynamic Assessment of Flight-Determined Subsonic Lift and Drag Characteristics of Seven Lifting-Body and Wing-Body Reentry Vehicle Configurations," NASA TP-2002-209032, 2002.

[2] Faget, M. A., Garland, B. J., and Buglia, J. J., "Preliminary Studies of Manned Satellites-Wingless Configuration: Nonlifting," NACA Conference on High-Speed Aerodynamics: A Compilation of the Papers Presented, NACA Ames Aeronautical Lab., Moffett Field, CA, 1958, pp. 19-33; also NASA TM-X-67369.

[3] Wong, T. J., Hermach, C. A., Reller, J. O., Jr., and Tinling, B. E., "Preliminary Studies of Manned Satellites: Wingless Configurations: Lifting Body," NACA Conference on High-Speed Aerodynamics-A Compilation of the Papers Presented, NACA Ames Aeronautical Lab., Moffett Field, CA, 1958, pp. 35-44; also NASA TM-X-67369.

[4] Becker, J. V., "Preliminary Studies of Manned Satellites: Winged Configurations," NACA Conference on High-Speed Aerodynamics-A Compilation of the Papers Presented, NACA Ames Aeronautical Lab., Moffett Field, CA, 1958, pp. 45-57; also NASA TM-X-67369.

[5] Chapman, D. R., "Study of Motion and Heating for Entry Into Planetary Atmospheres," NACA Conference on High-Speed Aerodynamics: A Compilation of the Papers Presented, NACA Ames Aeronautical Lab., Moffett Field, CA, 1958, pp. 1-17; also NASA TM-X-67369.

[6] Love, E. S., "Introductory Considerations of Manned Reentry Orbital Vehicles," Joint Conference on Lifting Manned Hypervelocity and Reentry Vehicles: A Compilation of the Papers Presented, NASA Langley Research Center, Langley Field, VA, 1960, pp. 39-54; also NASA TM-X-67563.

[7] Dennis, D. H., and Edwards, G. G., "The Aerodynamic Characteristics of Some Lifting Bodies," Joint Conference on Lifting Manned Hypervelocity and Reentry Vehicles: A Compilation of the Papers Presented, NASA Langley Research Center, Langley Field, VA, 1960, pp. 103-119; also NASA TM-X-67563.

[8] Paulson, J. W., Shanks, R. E., and Johnson, J. L., "Low-Speed Flight Characteristics of Reentry Vehicles of the Glide-Landing Type," Joint Conference on Lifting Manned Hypervelocity and Reentry Vehicles: A Compilation of the Papers Presented, NASA Langley Research Center, Langley Field, VA, 1960, pp. 329-344; also NASA TM-X-67563.

[9] Reed, R. D., and Lister, D., "Wingless Flight: The Lifting Body Story," NASA SP-4220, 1997.

[10] Horton, V. W., Eldredge, R. C., and Klein, R. E., "Flight-Determined Low-Speed Lift and Drag Characteristics of the Lightweight M2-F1 Lifting Body," NASA TN-D-3021, 1965.

[11] Smith, H. J., "Evaluation of the Lateral-Directional Stability and Control Characteristics of the Lightweight M2-F1 Lifting Body at Low Speeds," NASA TN-D-3022, 1965.

[12] Pyle, J. S., and Swanson, R. H., "Lift and Drag Characteristics of the M2-F2 Lifting Body During Subsonic Gliding Flight, "NASA TM-X1431, 1967.

[13] Pyle, J. S., "Lift and Drag Characteristics of the HL-10 Lifting Body During Subsonic Gliding Flight," NASA TN-D-6263, 1971.

[14] Ash, L. G., "Flight Test and Wind Tunnel Performance Characteristics of the X-24A Lifting Body," U.S. Air Force Flight Test Center FTCTD-71-8, Edwards AFB, CA, June 1972.

[15] Anon., "Comparison of Flight Test and Wind Tunnel Performance Characteristics of the X-24B Research Aircraft," U.S. Air Force Flight Test Center, Rept. TR-76-10, Edwards AFB, CA, Apr. 1976.

[16] Anon., "Flight Test Results Pertaining to the Space Shuttlecraft," NASA TM-X-2101, 1970.

[17] Anon., "Aerodynamics of Hypersonic Lifting Vehicles," AGARD CP428, Apr. 1987.

[18] Kempel, R. W., Painter, W. D., and Thompson, M. O., "Developing and Flight Testing the HL-10 Lifting Body: A Precursor to the Space Shuttle," NASA RP-1332, 1994.

[19] Iliff, K. W., and Peebles, C. L., "From Runway to Orbit, Reflections of a NASA Engineer," Government Printing Office, NASA History Series, NASA SP-2004-4109, 2004.
[20] Matranga, G. J., "Analysis of X-15 Landing Approach and Flare Characteristics Determined from the First 30 Flights," NASA TN-D$1057,1961$.

[21] Matranga, G. J., and Armstrong, N. A., "Approach and Landing Investigation at Lift-Drag Ratios of 2 to 4 Utilizing a Straight-Wing Fighter Airplane," NASA TM-X-31, 1959.

[22] Saltzman, E. J., and Garringer, D. J., "Summary of Full-Scale Lift and Drag Characteristics of the X-15 Airplane," NASA TN-D-3343, 1966.

[23] Thompson, M. O., "Final Remarks and Future Plans," Flight Test Results Pertaining to the Space Shuttlecraft, NASA TM-X-2101, 1970, pp. $147-151$.

[24] Anon., "AFFTC Evaluation of the Space Shuttle Orbiter and Carrier Aircraft: NASA Approach and Landing Test," U.S. Air Force Flight Test Center, Rept. TR-78-14, Edwards AFB, CA, May 1978.

[25] Krienes, K., "The Elliptic Wing Based on the Potential Theory," NACA TM-971, 1941.

[26] Helmbold, H. B., "Der unverwundene Ellipsenflügel als tragende Fläche," Jahrbuch der Deutschen Luftfahrtforschung, R. Oldenbourg, Munich, 1942, pp. I111-I113.

[27] Polhamus, E. C., "A Simple Method of Estimating the Subsonic Lift and Damping in Roll of Sweptback Wings," NACA TN-1862, 1949.

[28] Jones, R. T., "Properties of Low-Aspect-Ratio Pointed Wings at Speeds Below and Above the Speed of Sound," NACA Rept. 835, 1946.

[29] Brown, C. E., and Michael, W. H., Jr., "On Slender Delta Wings with Leading-Edge Separation," NACA TN-3430, 1955.

[30] Allen, H. J., and Perkins, E. W., "A Study of Effects of Viscosity on Flow over Slender Inclined Bodies of Revolution,” NACA Rept. 1048, 1951.

[31] Hoerner, S. F., and Borst, H. V., Fluid-Dynamic Lift: Practical Information on Aerodynamic and Hydrodynamic Lift, L. A. Hoerner, Brick Town, NJ, 1985.

[32] Hoerner, S. F., Fluid-Dynamic Drag: Practical Information on Aerodynamic Drag and Hydrodynamic Resistance, L. A. Hoerner, Brick Town, NJ, 1965.

[33] Raymer, D. P., Aircraft Design: A Conceptual Approach, AIAA Education Series, AIAA, Washington, D.C., 1992.

[34] Stinton, D., The Design of the Aeroplane, Van Nostrand Rheinhold Co., New York, 1983.

[35] Loftin, L. K., Jr., "Subsonic Aircraft: Evolution and the Matching of Size to Performance," NASA RP-1060, 1980.

[36] Oswald, W. B., "General Formulas and Charts for the Calculation of Airplane Performance," NACA Rept. 408, 1932.

[37] Wendt, R. E., "A Method of Airplane Performance Calculation Applicable to Any Polar," Journal of the Aeronautical Sciences, Vol. 14, No. 4, Apr. 1947, pp. 243-250.

[38] Perkins, C. D., and Hage, R. E., Airplane Performance Stability and Control, Wiley, New York, 1949.

[39] Peterson, J. B., Jr., "A Comparison of Experimental and Theoretical Results for the Compressible Turbulent-Boundary-Layer Skin Friction with Zero Pressure Gradient," NASA TN-D-1795, 1963.

[40] Bertram, M. H., "Calculations of Compressible Average Turbulent Skin Friction," NASA TR-R-123, 1962.

[41] Pearson, H. A., and Beadle, D. E., "Flight Measurements by Various Methods of the Drag Characteristics of the XP-51 Airplane," NACA MR-L6F12, 1946.

[42] Beeler, D. E., Bellman, D. R., and Saltzman, E. J., "Flight Techniques for Determining Airplane Drag at High Mach Numbers," NACA TN$3821,1956$.

[43] Ware, G. M., "Low-Subsonic-Speed Static Stability of RightTriangular-Pyramid and Half-Cone Lifting Reentry Configurations," NASA TN-D-646, 1961.

[44] Stivers, L. S., Jr., and Levy, L. L., Jr., "Longitudinal Force and Moment Data at Mach Numbers from 0.60 to 1.40 for a Family of Elliptic Cones with Various Semiapex Angles," NASA TN-D-1149, 1961.

[45] Knapp, R. J., Jordan, G. H., and Johnson, W. E., "Fuselage Pressures Measured on the Bell X-1 Research Airplane in Transonic Flight," NACA RM-L53I15, 1953.

[46] Pyle, J. S., and Montoya, L. C., "Effect of Roughness of Simulated Ablated Material on Low-Speed Performance Characteristics of a Lifting-Body Vehicle," NASA TM-X-1810, 1969.

[47] Layton, G. P., Jr., "Interim Results of the Lifting-Body Flight-Test Program," NASA TM-X-1827, 1969.

[48] Anon., "Correlation of X-24B Flight and Wind Tunnel Pressure Data," U.S. Air Force Flight Dynamics Laboratory, Rept. TR-78-93, Sept. 1978.

[49] Saltzman, E. J., "Base Pressure Coefficients Obtained from the X-15 Airplane for Mach Numbers up to 6," NASA TN-D-2420, 1964.

[50] Phillips, W. P., Compton, H. R., and Findlay, J. T., "Base Drag Determination for STS Flights 1-5," AIAA Paper 83-2719, Nov. 1983. 
[51] Butsko, J. E., Carter, W. V., and Herman, W., "Development of Subsonic Base Pressure Prediction Methods," U.S. Air Force Flight Dynamics Laboratory, Rept. TR-65-157, Vol. 1, Aug. 1965.

[52] Whitmore, S. A., and Moes, T. R., "A Base Drag Reduction Experiment on the X-33 Linear Aerospike SR-71 Experiment (LASRE) Flight Program,” AIAA 99-0277, Jan. 1999.
[53] Saltzman, E. J., "Preliminary Full-Scale Power-Off Drag of the X-15 Airplane for Mach Numbers from 0.7 to 3.1," NASA TM-X-430, 1960.

P. Huseman Associate Editor 